\title{
The Spectroscopical and Microstructural Investigations of Nanoclusters and Micron-Sized Periodic Structures Created at the Surface of the Crystal and Amorphous Silica by Resonant $\mathrm{CO}_{2}$ Laser Irradiation
}

\author{
A.F.Mukhamedgalieva $^{1 \text { 1a }}$, A.M.Bondar ${ }^{1}$, I.M.Shvedov ${ }^{1}$, M.A.Kononov², V.B.Laptev ${ }^{3}$, N.N.Novikova ${ }^{3}$ \\ ${ }^{1}$ NITU MISiS.Moscow., Leninsky prospect 4, P.O. 119049, Moscow, Russia \\ 2 Institute of General Physics, Russian Academy of Sciences P.O. 119991, Moscow. Russia, Vavilov street 38, \\ ${ }^{3}$ Institute of Spectroscopy, Russian Academy of Sciences P.O. 142190, Moscow, Troitsk, Russia
}

\begin{abstract}
The action of pulsed $\mathrm{CO}_{2}$ laser radiation (pulse energy of $1 \mathrm{~J}$, pulse time of $70 \mathrm{~ns}$ ) at the surface of silica (crystal quartz and fused quartz ) have been investigated. By means of spectroscopical and microstructural investigations it has been established the appearrance of two kind of structures - periodical micron-sized structures with the period length close to wave length of $\mathrm{CO}_{2}$ laser irradiation and nanoclusters with size close to 50-100 nanometers.
\end{abstract}

Keywords: silica, $\mathrm{CO}_{2}$ laser, nanoclusters.

The formation of nanoclasters and micrometer sized periodical structures at the surface of silica (crystal quartz and fused quartz ) by action of pulsed $\mathrm{CO}_{2}$ laser radiation (pulse energy of $1 \mathrm{~J}$, pulse time of $70 \mathrm{~ns}$ ) have been investigated. The samples was irradiated by $\mathrm{CO}_{2}$ laser in two regimes single-mode (fluency of $40 \mathrm{~J} / \mathrm{cm}^{2}$ ) and multi-mode (fluency of $48 \mathrm{~J} / \mathrm{cm}^{2}$ ) and with two laser frequency -975 and $1076 \mathrm{~cm}^{-1}$.

The images of laser spots by means of the high resolution optical microscop and atomic force microscop have been made. The infrared (IR) reflection spectra and luminescence spectra of irradiated surface also have been recorded. It has been observed that by laser action on the surface of samples two kind of structures have appeared - periodical micron-sized structures with the period length close to wave length of $\mathrm{CO}_{2}$ laser irradiation and nanoclusters with size close to 50-100 nanometers. It has been observed that the relief depth of the periodic structures depends on laser pulses number.The maximal highness of nanoclusters at the resonant frequency of laser $\left(1076 \mathrm{~cm}^{-1}\right)$ has been observed. We believe that these nanoclusters consist mainly of silicon atoms that confirm the luminescence spectra of irradiated sample.

The IR reflection spectra for irradiated samples show the enhancement of reflectance in the region of laser frequency with the band width of $20 \mathrm{~cm}^{-1}$ for crystal quartz. More high enhancements have been found at the frequency of $1076 \mathrm{~cm}^{-1}$. It has established that the reflectance enhancement has an accumulating character, namely, dependence of this ones on the number of laser pulses incident upon the samples take place.

${ }^{a}$ Corresponding author: anel-mggu@mail.ru

This is an Open Access article distributed under the terms of the Creative Commons Attribution License 4.0, which permits unrestricted use, distribution, and reproduction in any medium, provided the original work is properly cited. 
We can make some conclusions:

1) The periodical micro-and nano-structures in a crystal and amorphous silica have appeared because of interference of incident waves and surface waves induced by incident waves in resonant absorption media. That structures arise because of the increase of ablation velocity at the maxims of standing waves.

2) Cumulative properties of periodical structures formation show us that the mainly ablation processes take place by laser action on silica surface.

3) Dependence of relief depth from frequency of laser action shows that microstructure formation connects with the resonant interaction of $\mathrm{CO}_{2}$ laser radiation with silicates. Intensive ablation in maxims of standing waves connects with the breaking of covalent oxygen - silicon bonds in region of laser action frequency what can be proved by IR spectra of irradiated samples [1].

\section{Reference}

1. A. Mukhamedgalieva, A. Bondar, Proc. SPIE, 2118-33, pp. 224-226 (1994) 